\title{
LIST OF FIGURES
}

No.

Title

$\underline{\text { Page }}$

1. Yield Curves; Target: $\mathrm{Pu}^{242}$; Flux: $7 \times 10^{13}$ neutrons/

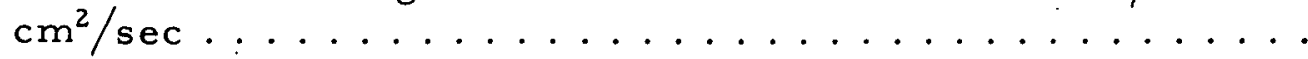

2. Yield Curves; Target: $P u^{242}$; Flux: $3 \times 10^{14}$ neutrons/ $\mathrm{cm}^{2} / \mathrm{sec} \ldots \ldots \ldots \ldots \ldots$

3. Yield Curves; Target: $\mathrm{Pu}^{242}$; Flux: $7 \times 10^{14}$ neutrons/ $\mathrm{cm}^{2} / \mathrm{sec} \ldots \ldots \ldots \ldots \ldots \ldots \ldots \ldots$

4. Yield Curves; Target: $\mathrm{Pu}^{242}$; Flux: $2 \times 10^{15}$ neutrons/

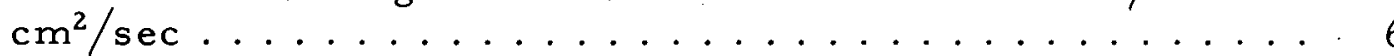

5. Yield Curves; Target: $\mathrm{Pu}^{242}$; Flux: $1 \times 10^{16}$ neutrons/ $\mathrm{cm}^{2} / \mathrm{sec} \ldots \ldots \ldots \ldots$

6. Yield Curves; Target: $\mathrm{Am}^{243}$; Flux: $7 \times 10^{13}$ neutrons/ $\mathrm{cm}^{2} / \mathrm{sec} \ldots \ldots \ldots \ldots$

7. Yield Curves; Target: Am ${ }^{243}$; Flux: $3 \times 10^{14}$ neutrons/ $\mathrm{cm}^{2} / \mathrm{sec} \ldots \ldots \ldots \ldots \ldots \ldots$

8. Yield Curves; Target: $\mathrm{Am}^{243}$; Fiux: $7 \times 10^{14}$ neutrons/ $\mathrm{cm}^{2} / \mathrm{sec} \ldots \ldots \ldots \ldots \ldots$

9. Yield Curves; Target: $\mathrm{Am}^{243}$; Flux: $2 \times 10^{15}$ neutrons/ $\mathrm{cm}^{2} / \mathrm{sec}$

10. Yield Curves; Target: $\mathrm{Am}^{243}$; Flux: $1 \times 10^{16}$ neutrons/ $\mathrm{cm}^{2} / \mathrm{sec} \ldots \ldots \ldots \ldots \ldots$

11. Yield Curves; Target: $\mathrm{Cm}^{244}$; Flux: $7 \times 10^{13}$ neutrons/ $\mathrm{cm}^{2} / \mathrm{sec}$

12. Yield Curves; Target: $\mathrm{Cm}^{244}$; Flux: $7 \times 10^{14}$ neutrons/ $\mathrm{cm}^{2} / \mathrm{sec}$

13. Yield Curves; Target: $\mathrm{Cm}^{244}$; Flux: $2 \times 10^{15}$ neutrons/ $\mathrm{cm}^{2} / \mathrm{sec}$

14. Yield Curves; Target: $\mathrm{Cm}^{246}$; Flux: $7 \times 10^{13}$ neutrons/ $\mathrm{cm}^{2} / \mathrm{sec} \ldots \ldots \ldots \ldots \ldots$ 


\section{DISCLAIMER}

This report was prepared as an account of work sponsored by an agency of the United States Government. Neither the United States Government nor any agency Thereof, nor any of their employees, makes any warranty, express or implied, or assumes any legal liability or responsibility for the accuracy, completeness, or usefulness of any information, apparatus, product, or process disclosed, or represents that its use would not infringe privately owned rights. Reference herein to any specific commercial product, process, or service by trade name, trademark, manufacturer, or otherwise does not necessarily constitute or imply its endorsement, recommendation, or favoring by the United States Government or any agency thereof. The views and opinions of authors expressed herein do not necessarily state or reflect those of the United States Government or any agency thereof. 


\section{DISCLAIMER}

Portions of this document may be illegible in electronic image products. Images are produced from the best available original document. 


\section{LIST OF FIGURES}

No.

Title

$\underline{\text { Page }}$

15. Yield Curves; Target: $\mathrm{Cm}^{246}$; Flux: $7 \times 10^{14}$ neutrons/.

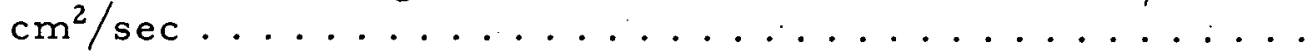

16. Yield Curves; Target: $\mathrm{Cm}^{246}$; Flux: $2 \times 10^{15}$ neutrons/

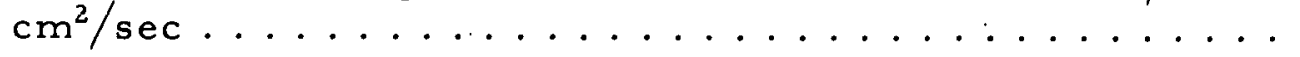

17. Yield Curves; Target: $\mathrm{Cm}^{246}$; Flux: $1 \times 10^{16}$ neutrons/

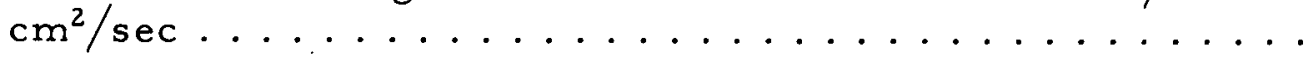

18. Yield Curves; Target: $\mathrm{Cf}^{252}$; Flux: $7 \times 10^{13}$ neutrons/

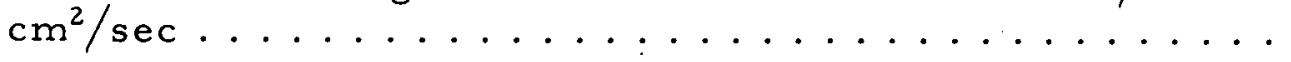

19. Yield Curves; Target: $\mathrm{Cf}^{252}$; Flux: $3 \times 10^{14}$ neutrons/

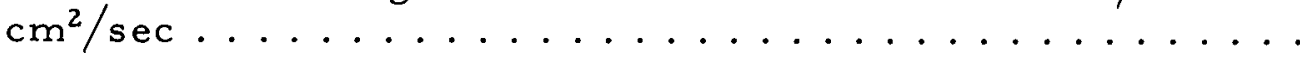

20. Yield Curves; Target: $\mathrm{Cf}^{252}$; Flux: $7 \times 10^{14}$ neutrons/

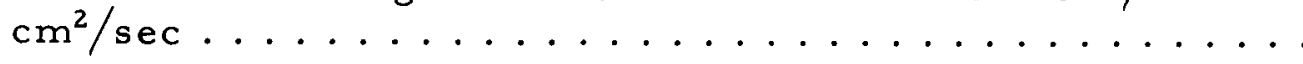

21. Yield Curves; Target: $\mathrm{Cf}^{252}$; Flux: $2 \times 10^{15}$ neutrons/

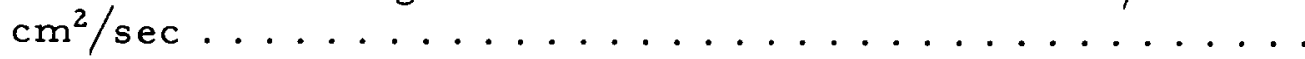

\section{LIST OF TABLES}

No. $\underline{\text { Title }}$

I. Available Yield Data...................

II. Maximum Obtainable Yields of Products from Heavy-element Targets as a Function of Flux ......... 


\section{ADDITIONAL YIELD DATA FOR TRANSPLUTONIUM ELEMENT PRODUCTION IN THERMAL REACTORS}

John Milsted, E. S. Macias, L. J. Basile, R. W. Anderson, and D. C. Stewart

Over the last several years the Argonne Heavy Element Chemistry Group has published several reports ${ }^{1,2,3}$ presenting the calculated yields of the heaviest nuclides as formed in various transneptunium targets by multiple neutron capture in a reactor. The ready availability of the computer programs used in this work has made it worthwhile to determine whether optimum target-flux combinations exist for obtaining a maximum yield of any particular heavy-element product. (It has been observed previously ${ }^{2}$ that an optimum flux range exists for producing $\mathrm{Cm}^{242}$ from $\mathrm{Am}^{241}$.) Extension of the earlier calculations to a broader range of fluxes also supplements the earlier work in providing reference material usable with a variety of reactors. Table I indicates the present availability of the heavy-nuclide yield curves that have been prepared under this program.

TABLE I. Available Yield Data

\begin{tabular}{lccccccc}
\hline & \multicolumn{7}{c}{ Flux (neutrons $/ \mathrm{cm}^{2} / \mathrm{sec}$ ) } \\
\cline { 2 - 7 } Target & $3 \times 10^{13}$ & $7 \times 10^{13}$ & $3 \times 10^{14}$ & $7 \times 10^{14}$ & $2 \times 10^{15}$ & $5 \times 10^{15}$ & $1 \times 10^{16}$ \\
\hline $\mathrm{Pu}^{239}$ & $(1)\left[4 \times 10^{13}\right]$ & - & $(1)$ & - & - & - & - \\
$\mathrm{Pu}^{242}$ & - & $(4)$ & $(4)$ & $(4)$ & $(3)$ & $(1)$ & $(3)$ \\
$\mathrm{Am}^{241}$ & $(2)$ & $(2)$ & $(1)$ & $(2)$ & $(2)$ & $(2)$ & $(2)$ \\
$\mathrm{Am}^{243}$ & - & $(4)$ & $(4)$ & $(4)$ & $(3)$ & $(1)$ & $(3)$ \\
$\mathrm{Cm}^{244}$ & - & $(4)$ & $(1)$ & $(4)$ & $(3)$ & $(1)$ & $(1)$ \\
$\mathrm{Cm}^{246}$ & - & $(4)$ & $(1)$ & $(4)$ & $(4)$ & $(1)$ & $(4)$ \\
$\mathrm{Cm}^{248}$ & - & - & $(1)$ & - & - & $(1)$ & - \\
$\mathrm{Cf}^{252}$ & - & $(4)$ & $(4)$ & $(4)$ & $(3)$ & $(1)$ & $(1)$ \\
\hline
\end{tabular}

$(1)_{\text {Ref. } 1 .}$.

(2) Ref. 2 .

(3) This report. Calculated by R. W. Anderson in preparing Ref. 3 but not previously published. Yields are given in terms of grams per gram of original target, rather than as atoms per original atom as in the other curves.

(4) This report. New calculations. 
The assumed build-up path, cross sections, and half-life data used are summarized in the earlier reports. They are essentially the same through the series, with but few minor variations. Computations for Refs. 1-3 were all made on the IBM-1620(II) computer, utilizing programs based either on the Bateman equations ${ }^{4}$ or on numerical integration, as described in the earlier reports. The numerical-integration approach was used exclusively for the present work, new programs being written to take advantage of the greater computing speed of the CDC-3600 computer. The yield data obtained on this instrument were adapted by a "converter" program to permit direct graphing of the yield curves (Figs. I-2l) by accessory equipment associated with the IBM-1620 machine.

Table II presents the maximum yield data for heavy-element nuclides as produced from various targets in a range of reactor fluxes. The products chosen were those of most interest to the chemist, usually the longest-lived species accessible by reactor production. Blanks in the table indicate that calculations were not carried out far enough on the time scale for the point of maximum yield to be seen.

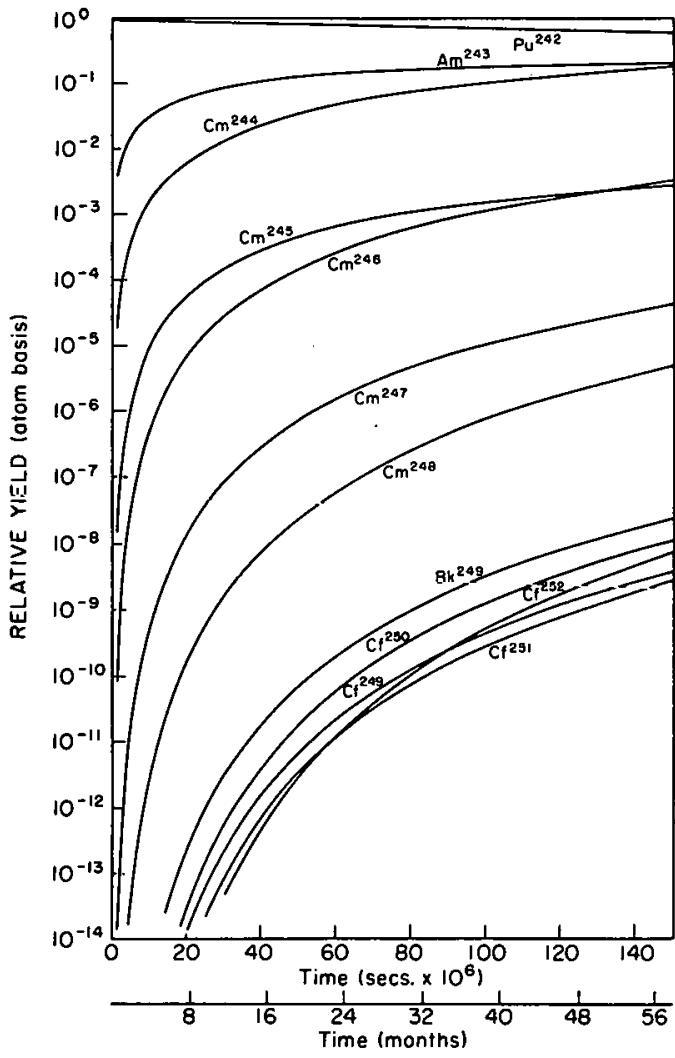

Fig. 1. Yield Curves; Target: $\mathrm{Pu}^{242}$; Flux: $7 \times 10^{13}$ neutrons $/ \mathrm{cm}^{2} / \mathrm{sec}$

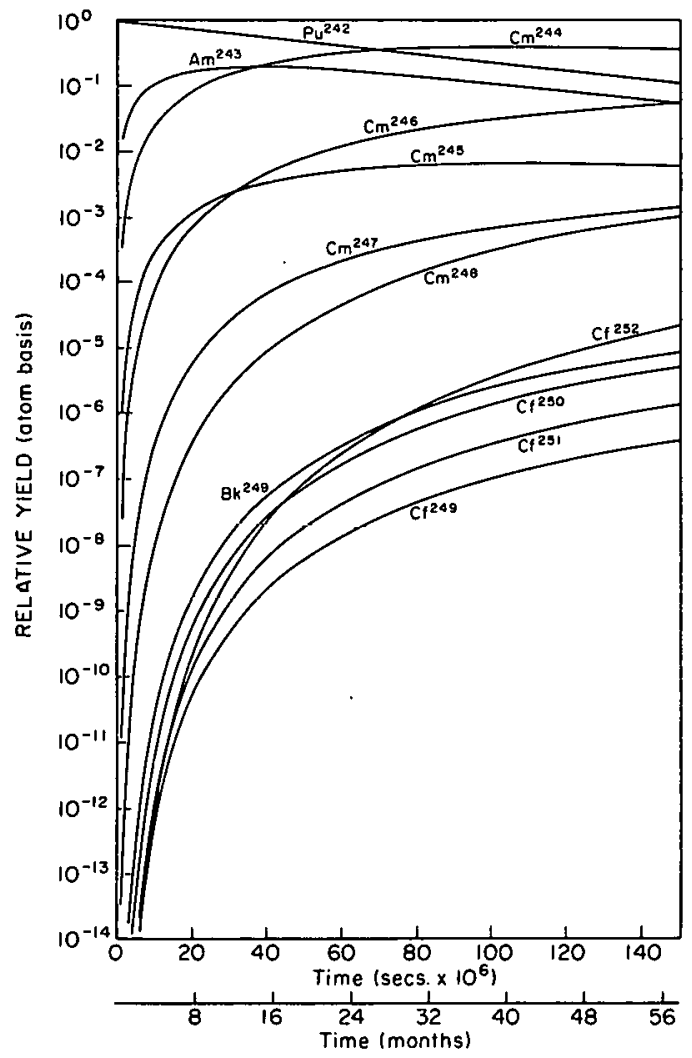

Fig. 2. Yield Curves; Target: $\mathrm{Pu}^{242}$; Flux: $3 \times 10^{14}$ neutrons $/ \mathrm{cm}^{2} / \mathrm{sec}$ 


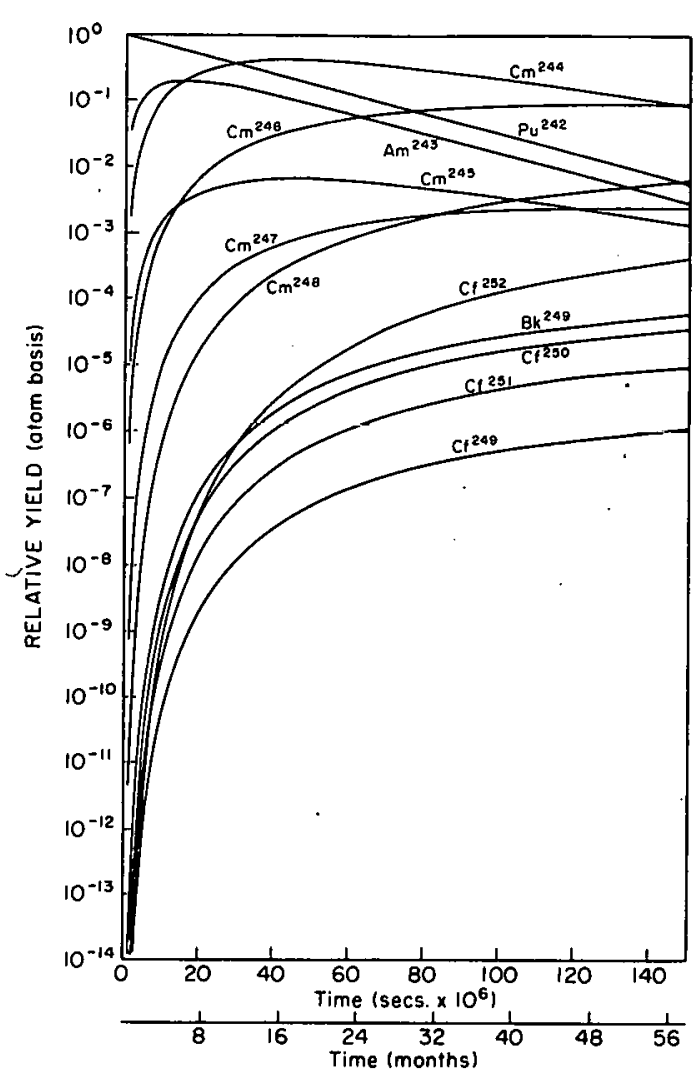

Fig. 3. Yield Curves; Target: $\mathrm{Pu}^{242}$; Flux: $7 \times 10^{14}$ neutrons $/ \mathrm{cm}^{2} / \mathrm{sec}$

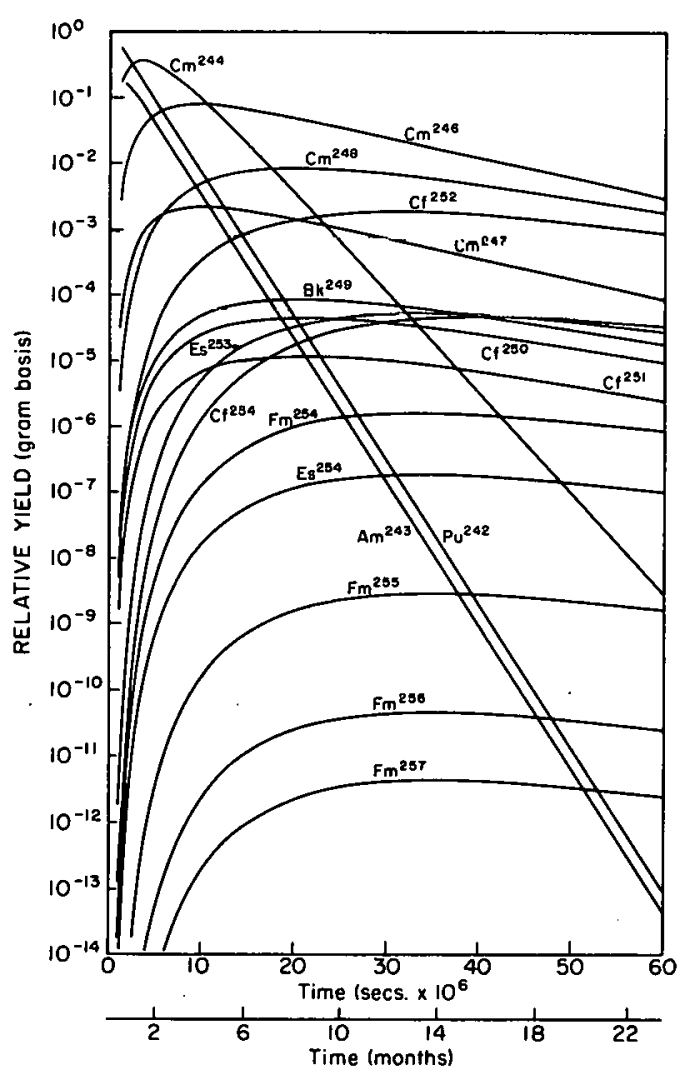

Fig. 5. Yield Curves; Target: $\mathrm{Pu}^{242}$; Flux: $1 \times 10^{16}$ neutrons $/ \mathrm{cm}^{2} / \mathrm{sec}$

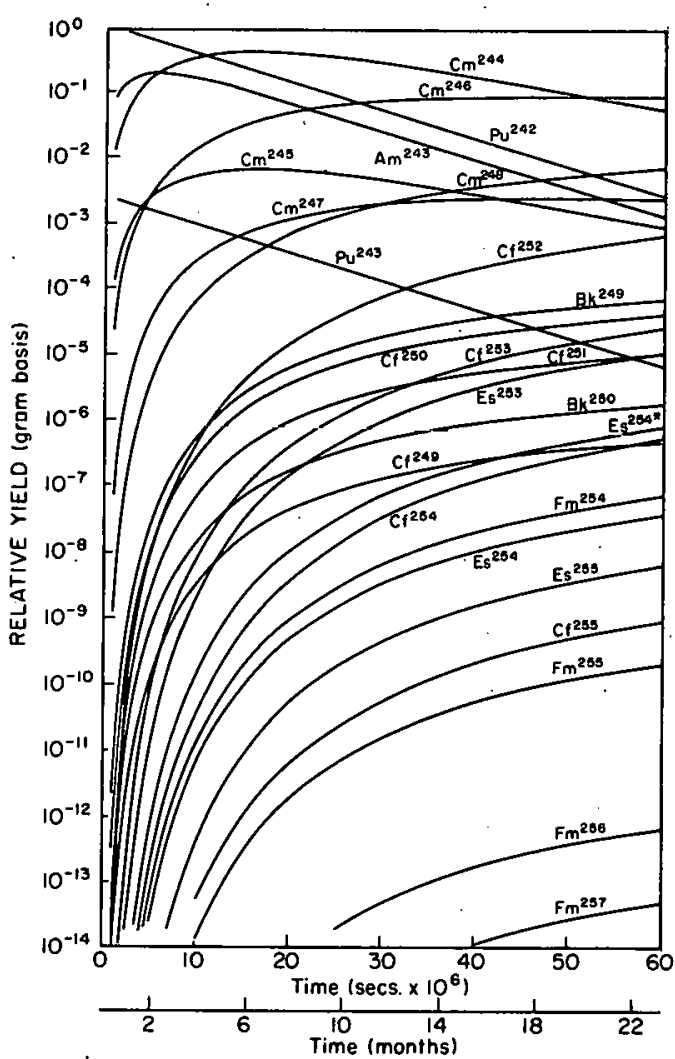

Fig. 4. Yield Curves; Target: $\mathrm{Pu}^{242}$; Flux: $2 \times 10^{15}$ neutrons $/ \mathrm{cm}^{2} / \mathrm{sec}$

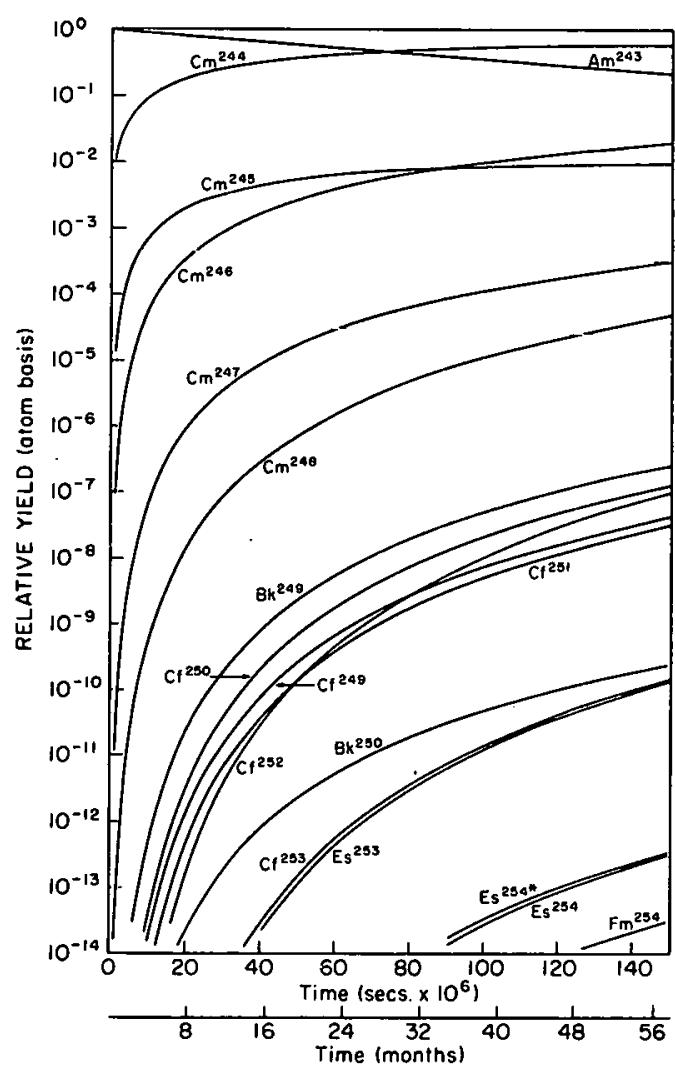

Fig. 6. Yield Curves; Target: $\mathrm{Am}^{243}$; Flux: $7 \times 10^{13}$ neutrons $/ \mathrm{cm}^{2} / \mathrm{sec}$ 


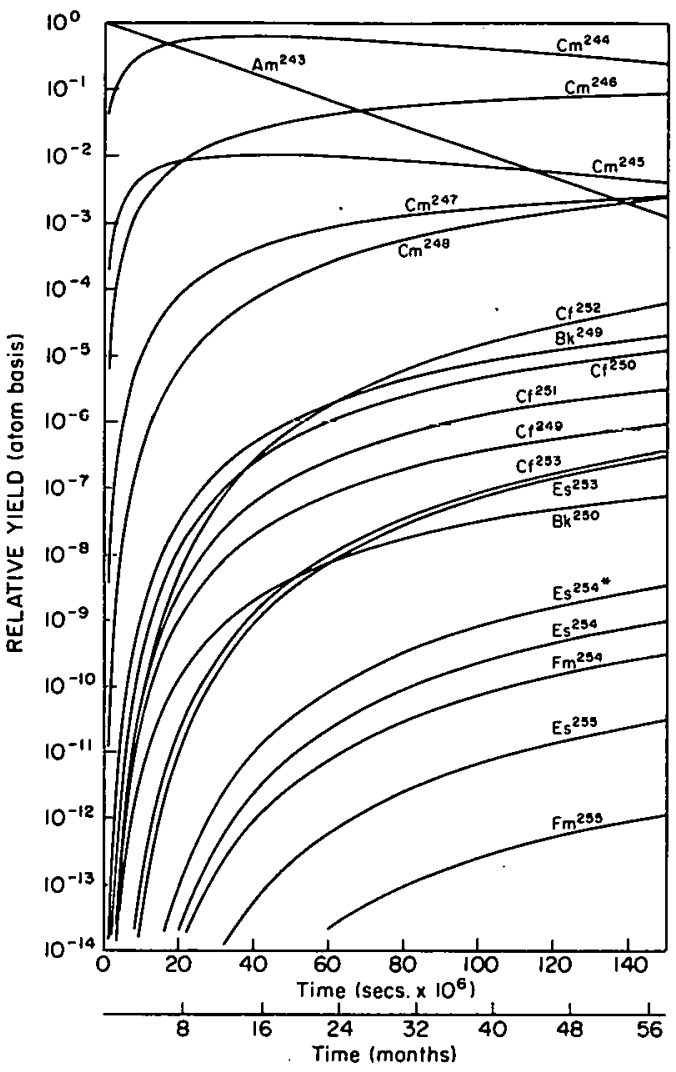

Fig. 7. Yield Curves; Target: $\mathrm{Am}^{243}$; Flux: $3 \times 10^{14}$ neutrons $/ \mathrm{cm}^{2} / \mathrm{sec}$

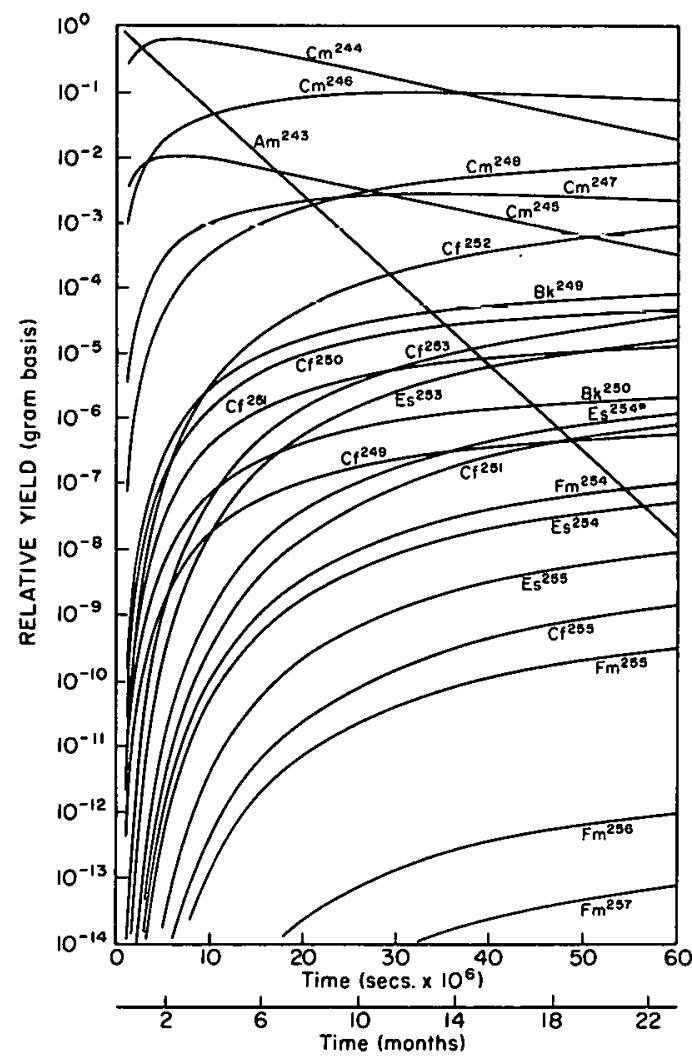

Fig. 9. Yield Curves; Target: $\mathrm{Am}^{243}$; Flux: $2 \times 10^{15}$ neutrons $/ \mathrm{cm}^{2} / \mathrm{sec}$

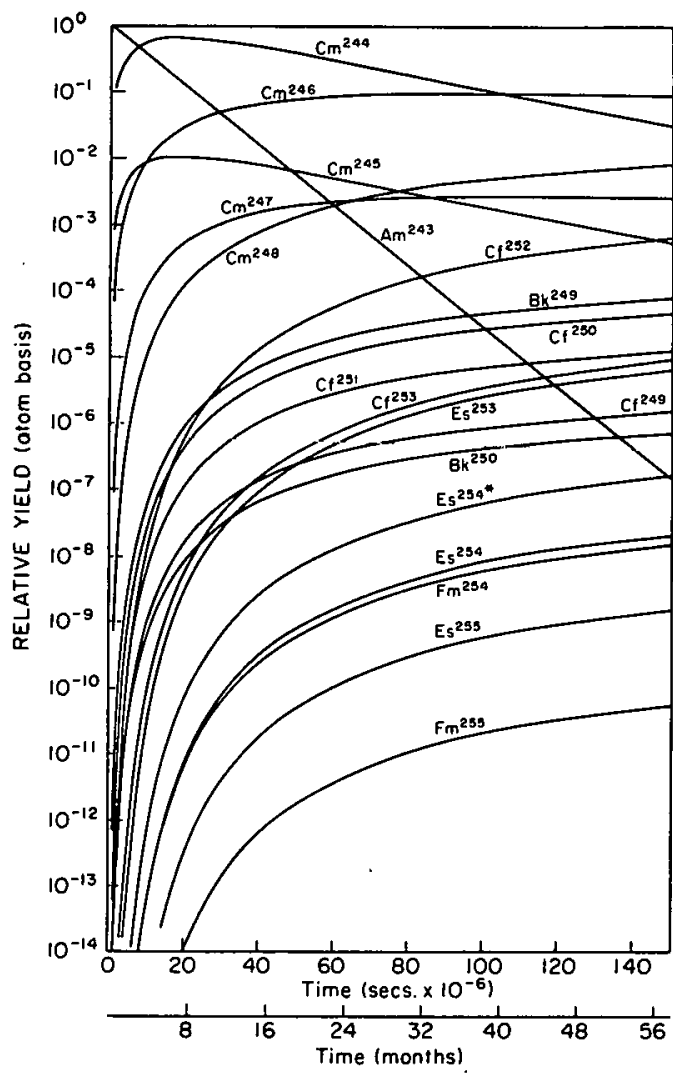

Fig. 8. Yield Curves; Target: $\mathrm{Am}^{243}$; Flux: $7 \times 10^{14}$ neutrons $/ \mathrm{cm}^{2} / \mathrm{sec}$

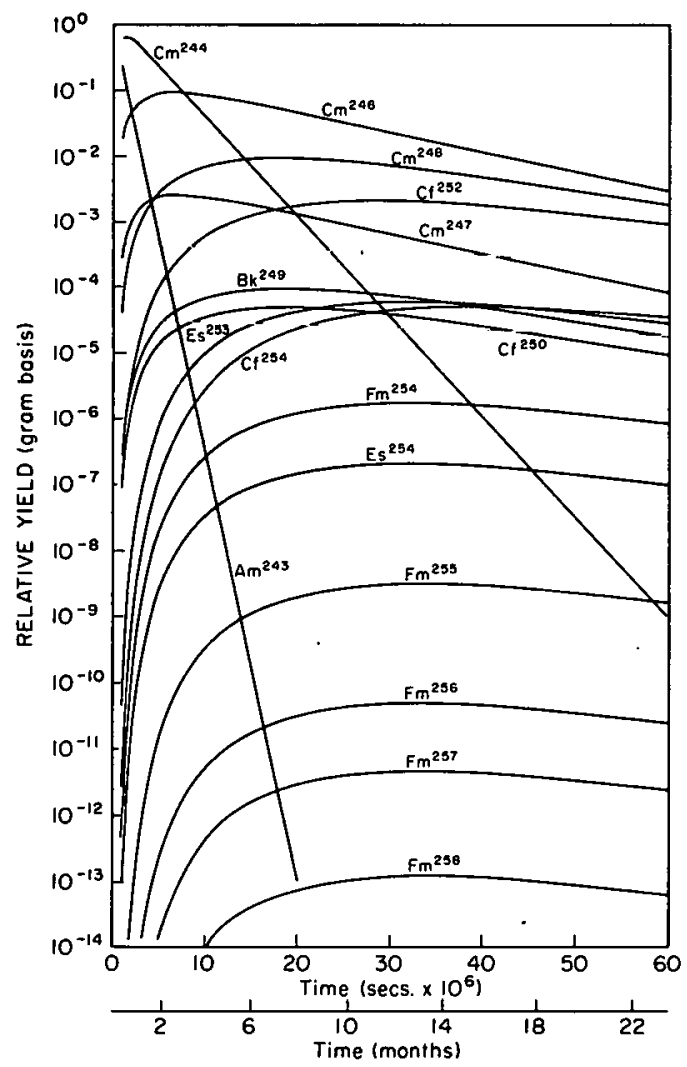

Fig. 10. Yield Curves; Target: $\mathrm{Am}^{243}$; Flux: $1 \times 10^{16}$ neutrons $/ \mathrm{cm}^{2} / \mathrm{sec}$ 


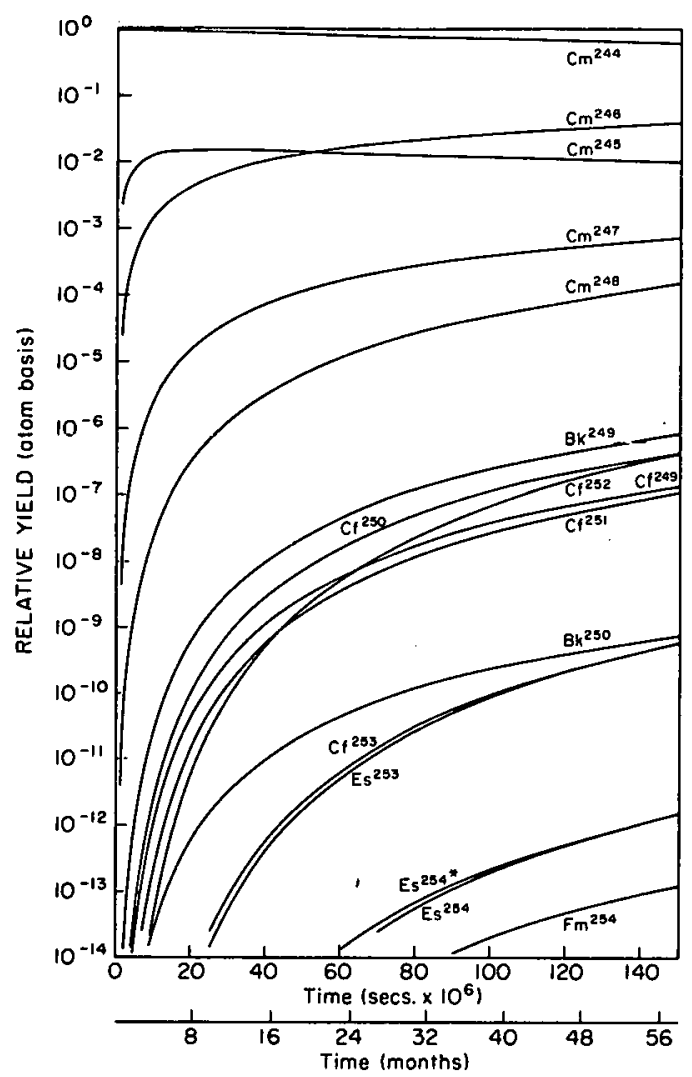

Fig. 11. Yield Curves; Target; $\mathrm{Cm}^{244}$; Flux: $7 \times 10^{13}$ neutrons $/ \mathrm{cm}^{2} / \mathrm{sec}$

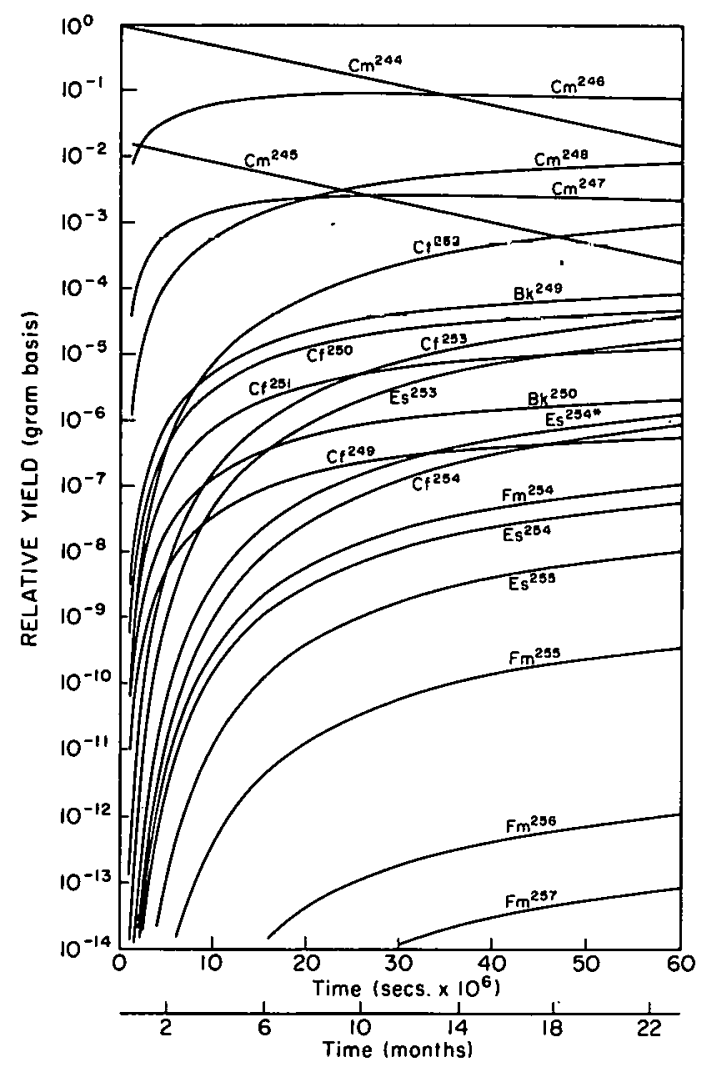

Fig. 13. Yield Curves; Target: $\mathrm{Cm}^{244}$; Flux: $2 \times 10^{15}$ neutrons $/ \mathrm{cm}^{2} / \mathrm{sec}$

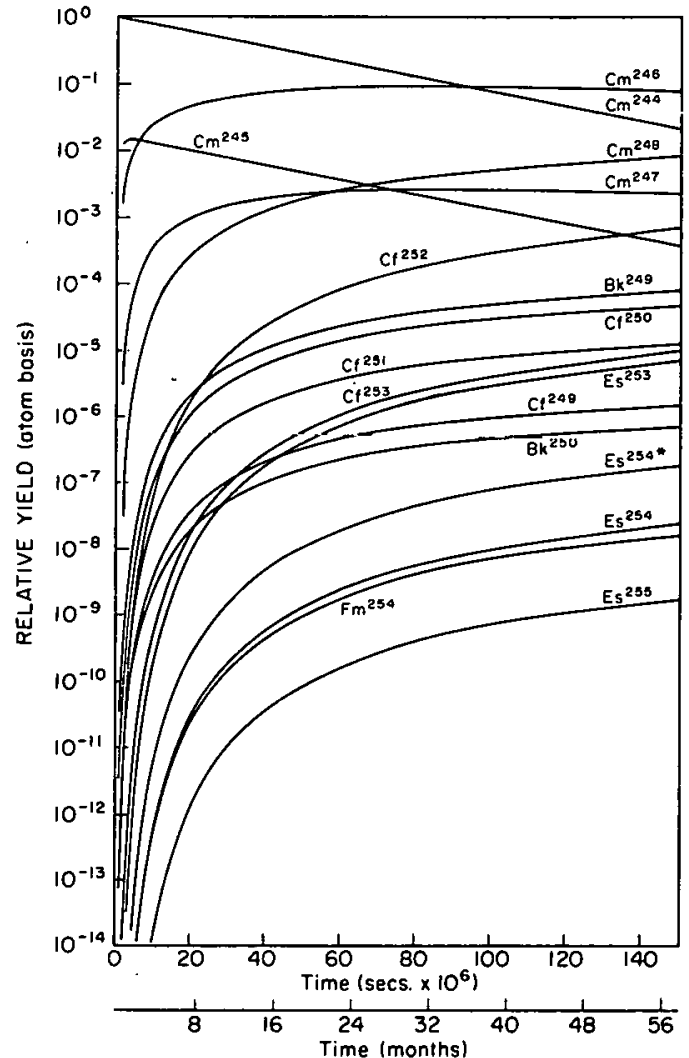

Fig. 12. Yield Curves; Target: $\mathrm{Cm}^{244}$; Flux: $7 \times 10^{14}$ neutrons $/ \mathrm{cm}^{2} / \mathrm{sec}$

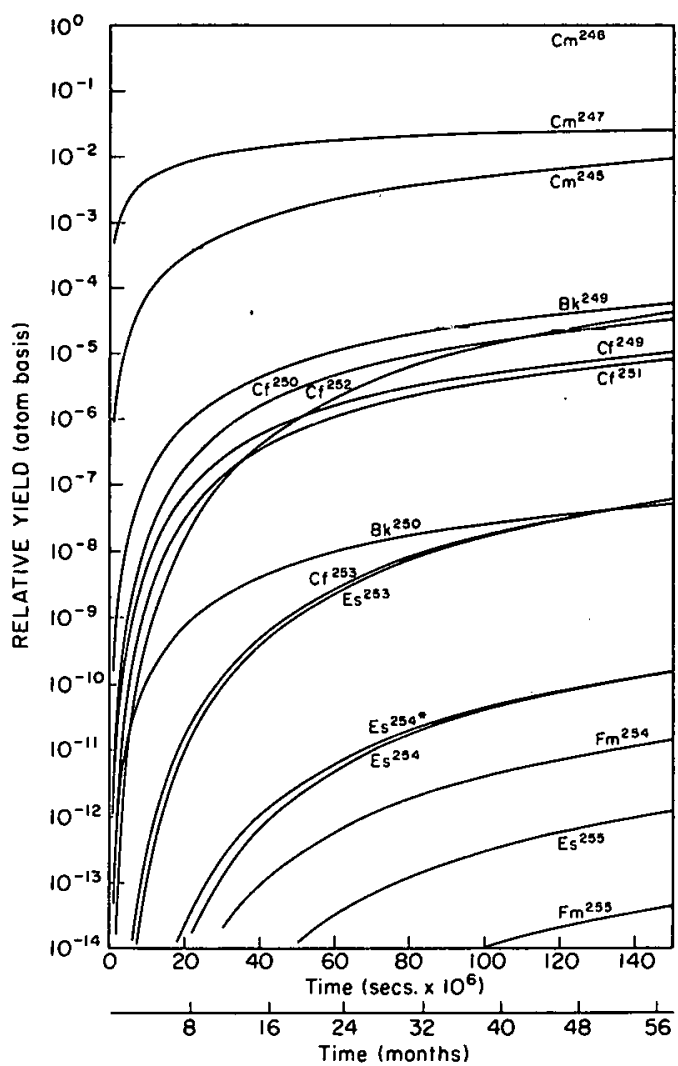

Fig. 14. Yield Curves; Target: $\mathrm{Cm}^{246}$; Flux: $7 \times 10^{13}$ neutrons $/ \mathrm{cm}^{2} / \mathrm{sec}$ 


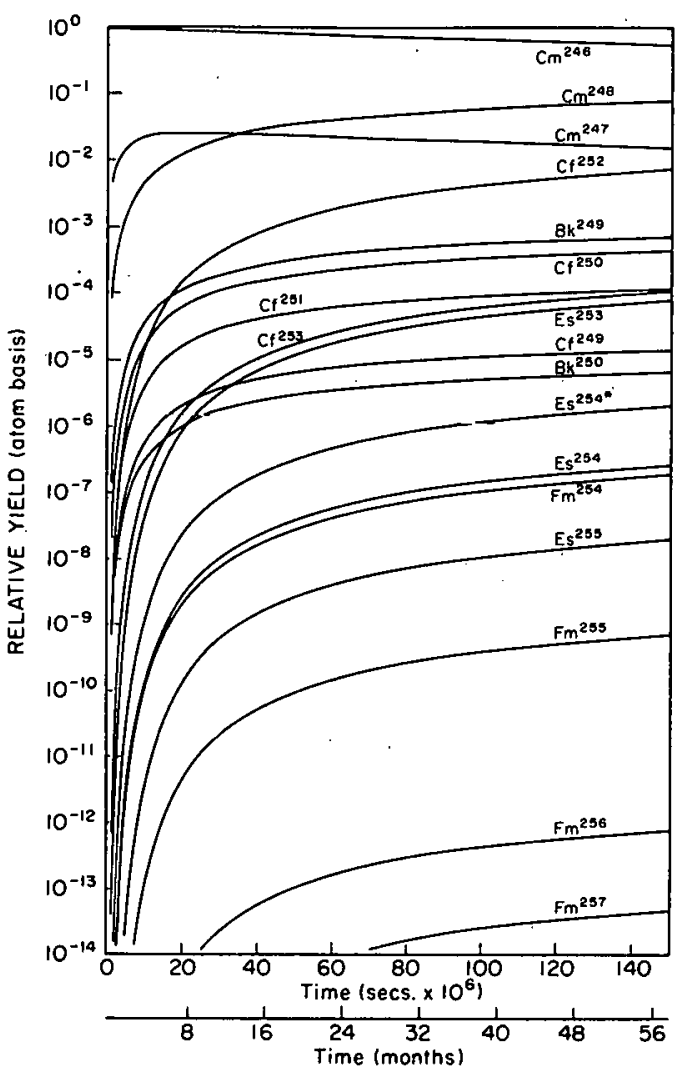

Fig. 15. Yield Curves; Target: $\mathrm{Cm}^{246}$; Flux: $7 \times 10^{14}$ neutrons $/ \mathrm{cm}^{2} / \mathrm{sec}$.

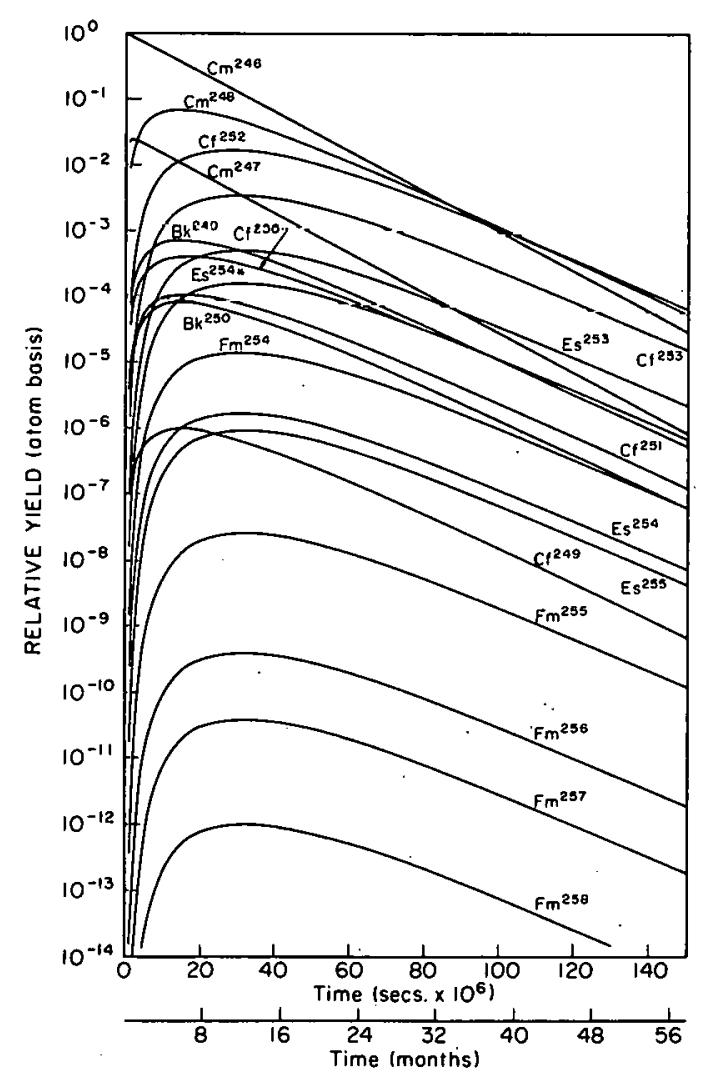

Fig. 17. Yield Curves; Target: $\mathrm{Cm}^{246}$; Flux: $1 \times 10^{16}$ neutrons $/ \mathrm{cm}^{2} / \mathrm{sec}$

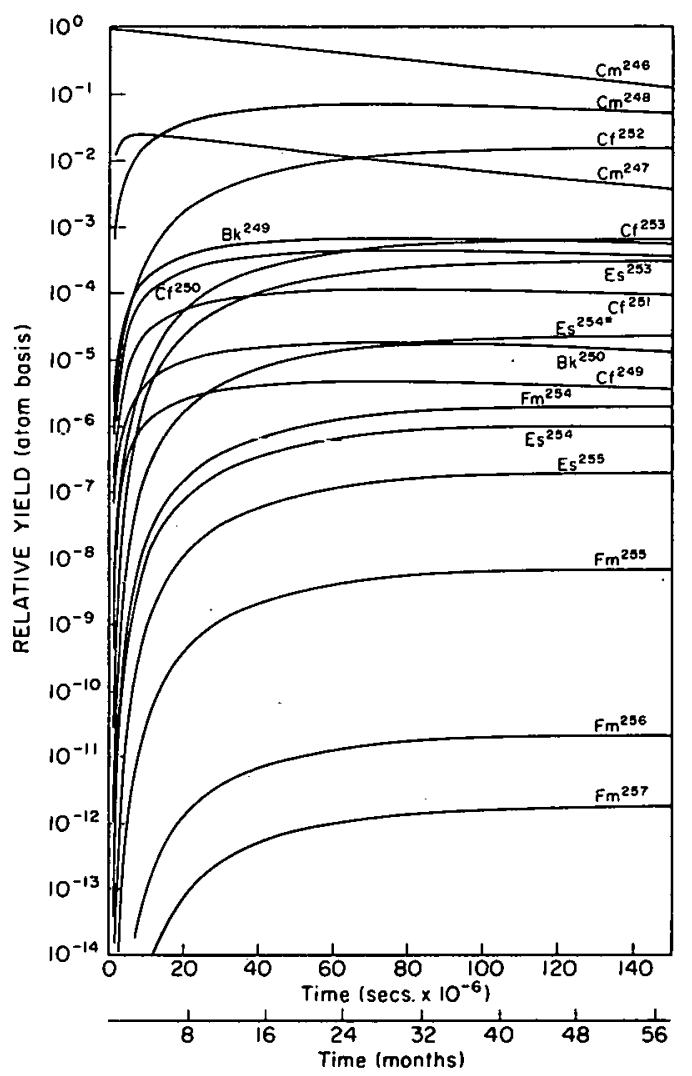

Fig. 16. Yield Curves; Target: $\mathrm{Cm}^{246}$; Flux: $2 \times 10^{15}$ neutrons $/ \mathrm{cm}^{2} / \mathrm{sec}$

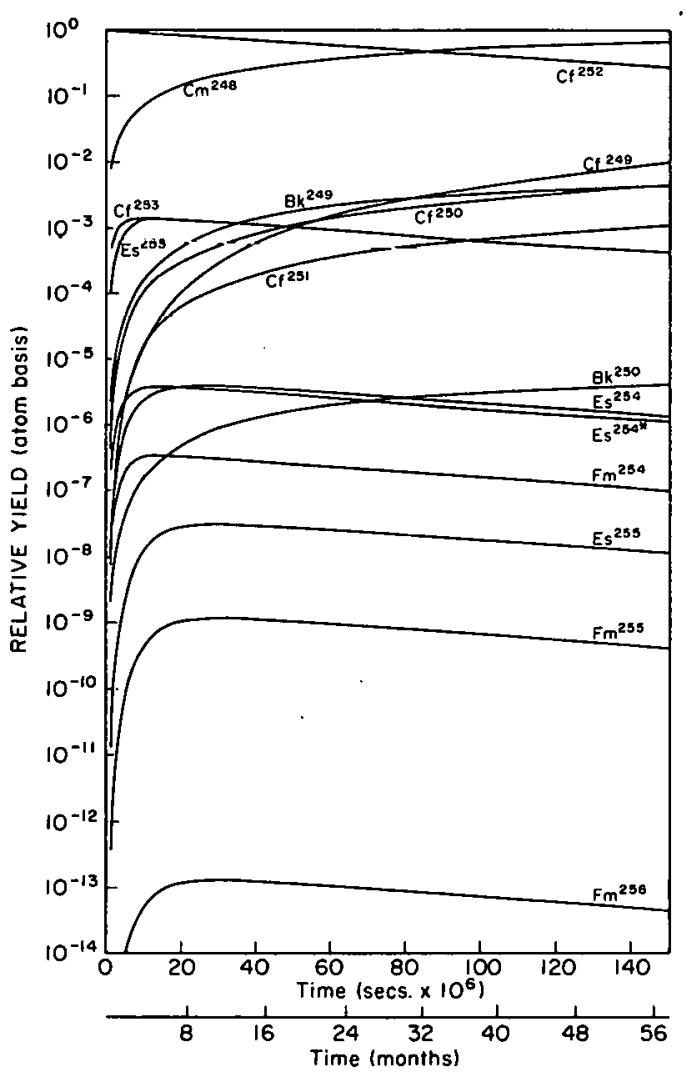

Fig, 18. Yield Curves; Target: $\mathrm{Cf}^{252}$; Flux; $7 \times 10^{13}$ neutrons $/ \mathrm{cm}^{2} / \mathrm{sec}$ 


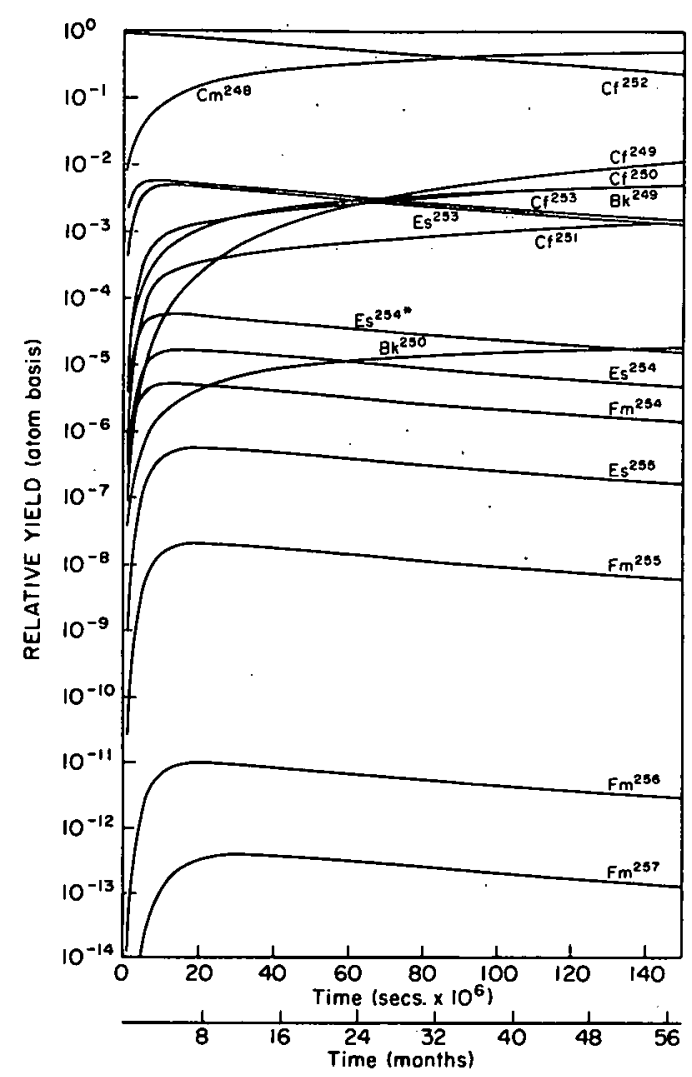

Fig. 19. Yield Curves; Target: $\mathrm{Cf}^{252}$; Flux: $3 \times 10^{14}$ neutrons $/ \mathrm{cm}^{2} / \mathrm{sec}$

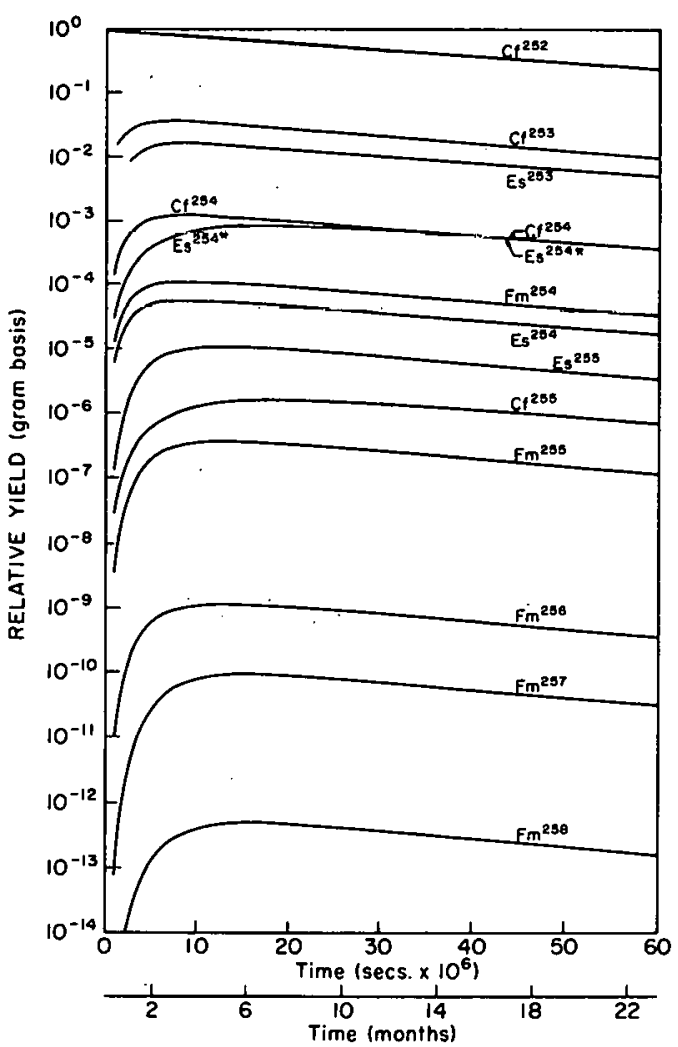

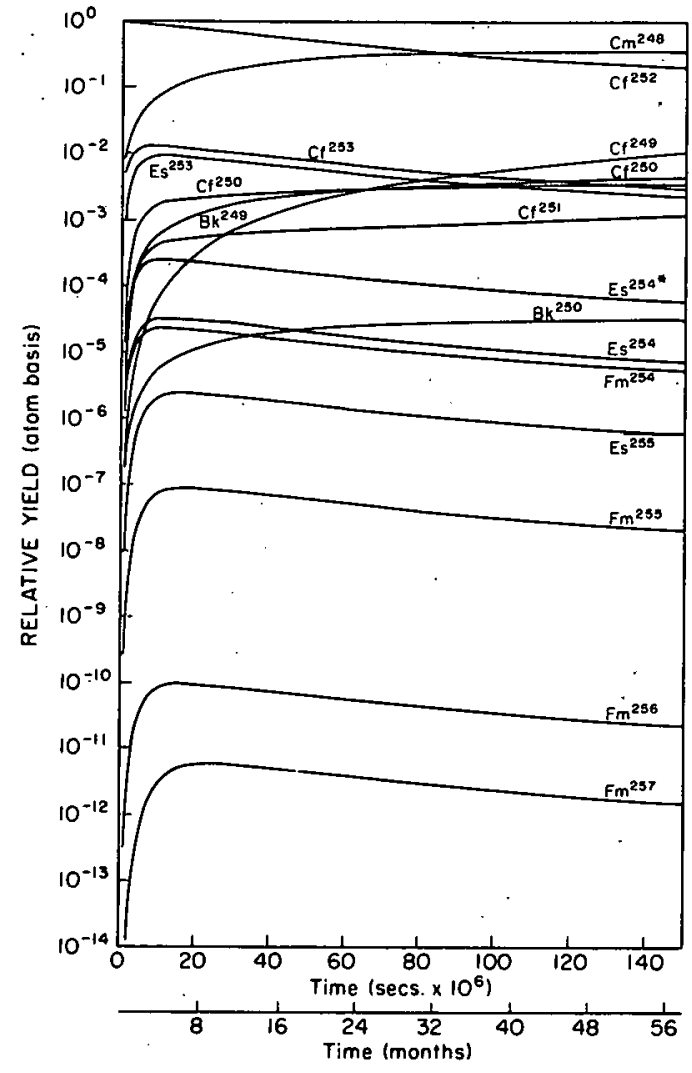

Fig. 20. Yield Curves; Target: $\mathrm{Cf}^{252}$; Flux: $7 \times 10^{14}$ neutrons $/ \mathrm{cm}^{2} / \mathrm{sec}$
Fig. 21

Yield Curves; Target: $\mathrm{Cf}^{252}$; Flux: $2 \times 10^{15}$ neutrons $/ \mathrm{cm}^{2} / \mathrm{sec}$ 
TABLE II. Maximum Obtainable Yields of Products from Heavy-element Targets as a Function of Flux

\begin{tabular}{|c|c|c|c|c|c|c|c|c|}
\hline \multirow{2}{*}{$\begin{array}{l}\text { Nuclide } \\
\text { Sought }\end{array}$} & \multirow[b]{2}{*}{ Target } & & \multicolumn{6}{|c|}{ Maximum Obtainable Relative Yield at Flux of: } \\
\hline & & & $7 \times 10^{13}$ & $3 \times 10^{14}$ & $7 \times 10^{14}$ & $2 \times 10^{15}$ & $5 \times 10^{15}$ & $1 \times 10^{16}$ \\
\hline $\mathrm{Am}^{243}$ & $\mathrm{Pu}^{242}$ & $\begin{array}{l}\text { Maximum yield } \\
\text { Time }^{\circ}\end{array}$ & - & $\begin{array}{r}0.191 \\
35 .\end{array}$ & $\begin{array}{r}0.191 \\
16\end{array}$ & $\begin{array}{r}0.188 \\
5\end{array}$ & $\begin{array}{r}0.185 \\
2.2\end{array}$ & $\begin{array}{r}0.178 \\
1.1\end{array}$ \\
\hline \multirow[t]{2}{*}{$\mathrm{Cm}^{244}$} & $\mathrm{Pu}^{242}$ & $\begin{array}{l}\text { Maximum yield } \\
\text { Time }^{\circ}\end{array}$ & $\cdot$ & $\begin{array}{r}0.391 \\
100\end{array}$ & $\begin{array}{r}0.402 \\
45\end{array}$ & $\begin{array}{r}0.404 \\
16\end{array}$ & $\begin{array}{c}0.396 \\
6\end{array}$ & $\begin{array}{r}0.383 \\
3.2\end{array}$ \\
\hline & $A m^{243}$ & $\begin{array}{l}\text { Maximum yield } \\
\text { Time }^{\circ}\end{array}$ & - & $\begin{array}{r}0.622 \\
40\end{array}$ & $\begin{array}{r}0.632 \\
18\end{array}$ & $\begin{array}{r}0.638 \\
6\end{array}$ & $\begin{array}{r}0.640 \\
2.5\end{array}$ & $\begin{array}{r}0.640 \\
1.2\end{array}$ \\
\hline \multirow[t]{3}{*}{$\mathrm{Cm}^{246}$} & $\mathrm{Pu}^{242}$ & $\begin{array}{l}\text { Maximum yield } \\
\text { Time }^{\circ}\end{array}$ & - & - & $\begin{array}{c}0.0832 \\
130\end{array}$ & $\begin{array}{c}0.0845 \\
45\end{array}$ & $\begin{array}{c}0.0833 \\
18\end{array}$ & $\begin{array}{c}0.0801 \\
10\end{array}$ \\
\hline & $A m^{243}$ & $\begin{array}{l}\text { Maximum yield } \\
\text { Time }^{\circ}\end{array}$ & - & - & $\begin{array}{c}0.0891 \\
90\end{array}$ & $\begin{array}{c}0.0914 \\
32\end{array}$ & $\begin{array}{c}0.0919 \\
14\end{array}$ & $\frac{0.0922}{7}$ \\
\hline & $\mathrm{Cm}^{244}$ & $\begin{array}{l}\text { Maximum yield } \\
\text { Time }^{\circ}\end{array}$ & - & $\begin{array}{c}0.0858 \\
180\end{array}$ & $\begin{array}{c}0.0899 \\
80\end{array}$ & $\begin{array}{c}0.0920 \\
30\end{array}$ & $\begin{array}{c}0.0927 \\
11\end{array}$ & $\begin{array}{c}0.0930 \\
6\end{array}$ \\
\hline \multirow[t]{4}{*}{$\mathrm{Cm}^{248}$} & $\mathrm{Pu}^{242}$ & $\begin{array}{l}\text { Maximum yield } \\
\text { Time }^{\circ}\end{array}$ & - & - & - & - & $\begin{array}{l}0.00878 \\
40\end{array}$ & $\begin{array}{l}0.00849 \\
20\end{array}$ \\
\hline & $A m^{243}$ & $\begin{array}{l}\text { Maximum yield } \\
\text { Time? }\end{array}$ & - & - & - & - & $\begin{array}{l}0.00923 \\
35\end{array}$ & $\begin{array}{l}0.00927 \\
18\end{array}$ \\
\hline & $\mathrm{Cm}^{244}$ & $\begin{array}{l}\text { Maximum yield } \\
\text { Time }^{\circ}\end{array}$ & - & - & - & - & $\begin{array}{l}0.00925 \\
35\end{array}$ & $\begin{array}{c}0.00926 \\
18\end{array}$ \\
\hline & $\mathrm{Cm}^{246}$ & $\begin{array}{l}\text { Maximum yield } \\
\text { Time }^{\alpha}\end{array}$ & - & - & - & $\begin{array}{c}0.0709 \\
70\end{array}$ & $\begin{array}{c}0.0684 \\
30\end{array}$ & $\begin{array}{l}0.0691 \\
14\end{array}$ \\
\hline \multirow[t]{4}{*}{$B k^{249}$} & $\mathrm{Pu}^{242}$ & $\begin{array}{l}\text { Maximum yield } \\
\text { Time }^{\star}\end{array}$ & - & $\cdot$ & - & - & $\begin{array}{c}8.72 \times 10^{-5} \\
40\end{array}$ & $\begin{array}{c}8.46 \times 10^{-5} \\
20\end{array}$ \\
\hline & $\mathrm{Am}^{243}$ & $\begin{array}{l}\text { Maximum yield } \\
\text { Time }^{\star} \text {. }\end{array}$ & - & - & - & $\cdot$ & $\frac{9.17 \times 10^{-5}}{35}$ & $\begin{array}{c}9.23 \times 10^{-5} \\
18\end{array}$ \\
\hline & $\mathrm{Cm}^{244}$ & $\begin{array}{l}\text { Maximum yield } \\
\text { Time }^{a}\end{array}$ & - & - & - & - & $\begin{array}{c}9.19 \times 10^{-5} \\
35\end{array}$ & $\begin{array}{c}9.24 \times 10^{-5} \\
18\end{array}$ \\
\hline & $\mathrm{Cm}^{246}$ & $\begin{array}{l}\text { Maximum yield } \\
\text { Time }^{\circ}\end{array}$ & - & - & - & $\begin{array}{c}6.98 \times 10^{-4} \\
70\end{array}$ & $\begin{array}{c}6.80 \times 10^{-4} \\
30\end{array}$ & $\begin{array}{c}6.89 \times 10^{-4} \\
14\end{array}$ \\
\hline \multirow[t]{4}{*}{$\mathrm{Ct}^{252}$} & $\mathrm{Pu}^{242}$ & $\begin{array}{l}\text { Maximum yield } \\
\text { Time }\end{array}$ & $\cdot$ & - & $\cdot$ & - & $\begin{array}{l}0.00200 \\
60\end{array}$ & $\begin{array}{l}0.00192 \\
30\end{array}$ \\
\hline & $\mathrm{Am}^{243}$ & $\begin{array}{l}\text { Maximum yield } \\
\text { Time }^{\circ}\end{array}$ & - & - & - & - & $\begin{array}{l}0.00209 \\
60\end{array}$ & $\begin{array}{l}0.00208 \\
30\end{array}$ \\
\hline & $\mathrm{Cm}^{244}$ & $\begin{array}{l}\text { Maximum yield } \\
\text { Time }^{\circ}\end{array}$ & - & - & - & - & $\begin{array}{l}0.00208 \\
60\end{array}$ & $\begin{array}{l}0.00207 \\
30\end{array}$ \\
\hline & $\mathrm{Cm}^{246}$ & $\begin{array}{l}\text { Maximum yield } \\
\text { Time }^{\circ}\end{array}$ & - & - & - & $\begin{array}{c}0.0156 \\
120\end{array}$ & $\begin{array}{l}0.0154 \\
50\end{array}$ & $\begin{array}{l}0.0169 \\
25\end{array}$ \\
\hline \multirow[t]{6}{*}{$\begin{array}{l}\mathrm{Es}^{254} \\
480 \mathrm{~d}\end{array}$} & $\mathrm{Pu}^{212}$ & $\begin{array}{l}\text { Maximum yleld } \\
\text { Time }^{\star}\end{array}$ & $\cdot$ & - & - & - & $\begin{array}{c}1.77 \times 10^{-7} \\
60\end{array}$ & $\begin{array}{c}1.88 \times 10^{-7} \\
35\end{array}$ \\
\hline & $\mathrm{Am}^{243}$ & $\begin{array}{l}\text { Maximum yield } \\
\text { Time }^{\circ}\end{array}$ & - & - & - & - & $\begin{array}{c}1.87 \times 10^{-7} \\
60^{\circ}\end{array}$ & $\begin{array}{c}2.05 \times 10^{-7} \\
32\end{array}$ \\
\hline & $\mathrm{Cm}^{244}$ & $\begin{array}{l}\text { Maximum yield } \\
\text { Time }^{\circ}\end{array}$ & - & - & - & - & $\begin{array}{c}1.87 \times 10^{-7} \\
60\end{array}$ & $\begin{array}{c}2.05 \times 10^{-7} \\
32\end{array}$ \\
\hline & $\mathrm{Cm}^{246}$ & $\begin{array}{l}\text { Maximum yield } \\
\text { Time }^{\circ}\end{array}$ & $\cdot$ & - & - & $\begin{array}{c}1.04 \times 10^{-6} \\
120\end{array}$ & $\begin{array}{c}1.37 \times 10^{-6} \\
50\end{array}$ & $\begin{array}{c}1.67 \times 10^{-6} \\
30\end{array}$ \\
\hline & $\mathrm{Cm}^{248}$ & $\begin{array}{l}\text { Maximum yield } \\
\text { Time }^{\circ}\end{array}$ & - & $\begin{array}{c}9.54 \times 10^{-7} \\
180\end{array}$ & - & $\cdot$ & $\begin{array}{c}1.02 \times 10^{-5} \\
25\end{array}$ & - \\
\hline & $\mathrm{Cr}^{252}$ & $\begin{array}{l}\text { Maximum yield } \\
\text { Time }\end{array}$ & $\begin{array}{c}3.84 \times 10^{-6} \\
25\end{array}$ & $\begin{array}{c}1.66 \times 10^{-5} \\
14\end{array}$ & $\begin{array}{c}3.20 \times 10^{-5} \\
12\end{array}$ & $\begin{array}{c}5.46 \times 10^{-5} \\
8\end{array}$ & $\begin{array}{c}6.60 \times 10^{-5} \\
6\end{array}$ & $\begin{array}{c}6.42 \times 10^{-5} \\
5\end{array}$ \\
\hline \multirow[t]{6}{*}{$\mathrm{Fm}^{255}$} & $\mathrm{Pu}^{242}$ & $\begin{array}{l}\text { Maximum yield } \\
\text { Time }^{\circ}\end{array}$ & - & - & - & - & $\begin{array}{c}2.12 \times 10^{-9} \\
70\end{array}$ & $\begin{array}{c}2.87 \times 10^{-9} \\
35\end{array}$ \\
\hline & $\mathrm{Am}^{243}$ & $\begin{array}{l}\text { Maximum yield } \\
\text { Time }^{\circ}\end{array}$ & - & - & - & - & $\begin{array}{c}2.23 \times 10^{-9} \\
60\end{array}$ & $\begin{array}{c}3.10 \times 10^{-9} \\
32\end{array}$ \\
\hline & $\mathrm{Cm}^{244}$ & $\begin{array}{l}\text { Maximum yield } \\
\text { Time }^{\circ}\end{array}$ & - & - & - & - & $\begin{array}{c}2.23 \times 10^{-9} \\
60\end{array}$ & $\begin{array}{c}3.11 \times 10^{-9} \\
32\end{array}$ \\
\hline & $\mathrm{Cm}^{246}$ & $\begin{array}{l}\text { Maximum yield } \\
\text { Time } e^{\circ}\end{array}$ & - & $\cdot$ & - & $\begin{array}{c}7.02 \times 10^{-9} \\
130\end{array}$ & $\begin{array}{c}1.62 \times 10^{-8} \\
60\end{array}$ & $\begin{array}{c}2.53 \times 10^{-8} \\
30\end{array}$ \\
\hline & $\mathrm{Cm}^{248}$ & $\begin{array}{l}\text { Maximum yield } \\
\text { Time }^{\circ}\end{array}$ & - & $\begin{array}{c}1.22 \times 10^{-9} \\
180\end{array}$ & - & - & $\begin{array}{c}1.21 \times 10^{-7} \\
25\end{array}$ & - \\
\hline & $\mathrm{Cl}^{252}$ & $\begin{array}{l}\text { Maximum yield } \\
\text { Time }^{\circ}\end{array}$ & $\begin{array}{c}1.18 \times 10^{-9} \\
30\end{array}$ & $\begin{array}{c}2.08 \times 10^{-8} \\
18\end{array}$ & $\begin{array}{c}8.73 \times 10^{-8} \\
16\end{array}$ & $\begin{array}{c}3.53 \times 10^{-7} \\
12\end{array}$ & $\begin{array}{c}7.43 \times 10^{-7} \\
10\end{array}$ & $\begin{array}{c}9.27 \times 10^{-7} \\
7\end{array}$ \\
\hline
\end{tabular}


TABLE II (Contd.)

\begin{tabular}{|c|c|c|c|c|c|c|c|c|}
\hline \multirow{2}{*}{$\begin{array}{l}\text { Nuclide } \\
\text { Sought }\end{array}$} & \multirow[b]{2}{*}{ Target } & & \multicolumn{6}{|c|}{ Maximum Obtainable Relative Yield at Flux of: } \\
\hline & & & $7 \times 10^{13}$ & $3 \times 10^{14}$ & $7 \times 10^{14}$ & $2 \times 10^{15}$ & $5 \times 10^{15}$ & $1 \times 10^{16}$ \\
\hline \multirow[t]{6}{*}{$\mathrm{Fm}^{257}$} & $\mathrm{Pu}^{242}$ & $\begin{array}{l}\text { Maximum yield } \\
\text { Time }^{\circ}\end{array}$ & - & - & - & - & $\begin{array}{c}1.53 \times 10^{-12} \\
70\end{array}$ & $\begin{array}{c}4.27 \times 10^{-12} \\
35\end{array}$ \\
\hline & $\mathrm{Am}^{243}$ & $\begin{array}{l}\text { Maximum yield } \\
\text { Time }^{\circ}\end{array}$ & - & - & - & - & $\begin{array}{c}1.60 \times 10^{-12} \\
60\end{array}$ & $\begin{array}{c}4.63 \times 10^{-12} \\
35\end{array}$ \\
\hline & $\mathrm{Cm}^{244}$ & $\begin{array}{l}\text { Maximum yield } \\
\text { Time }^{\circ}\end{array}$ & - & - & - & - & $\begin{array}{c}1.61 \times 10^{-12} \\
60\end{array}$ & $\begin{array}{c}4.64 \times 10^{-12} \\
32\end{array}$ \\
\hline & $\mathrm{Cm}^{246}$ & $\begin{array}{l}\text { Maximum yield } \\
\text { Time }^{\circ}\end{array}$ & - & - & - & $\begin{array}{c}1.82 \times 10^{-12} \\
130\end{array}$ & $\begin{array}{c}1.16 \times 10^{-11} \\
50\end{array}$ & $\begin{array}{c}3.78 \times 10^{-11} \\
32\end{array}$ \\
\hline & $\mathrm{Cm}^{248}$ & $\begin{array}{l}\text { Maximum yield } \\
\text { Time }^{\alpha}\end{array}$ & - & - & - & - & $\begin{array}{c}8.79 \times 10^{-11} \\
30\end{array}$ & - \\
\hline & $\mathrm{Cf} 252$ & $\begin{array}{l}\text { Maximum yield } \\
\text { Time }^{*}\end{array}$ & $\begin{array}{c}1.74 \times 10^{-15} \\
45\end{array}$ & $\begin{array}{c}3.80 \times 10^{-13} \\
30\end{array}$ & $\begin{array}{c}5.71 \times 10^{-12} \\
22\end{array}$ & $\begin{array}{c}8.93 \times 10^{-11} \\
16\end{array}$ & $\begin{array}{c}5.34 \times 10^{-10} \\
10\end{array}$ & $\begin{array}{c}1.37 \times 10^{-9} \\
7\end{array}$ \\
\hline \multirow[t]{2}{*}{$M^{259}$} & $\mathrm{Cm}^{248}$ & $\begin{array}{l}\text { Maximum yield } \\
\text { Time }^{\circ}\end{array}$ & - & - & - & - & $\begin{array}{c}3.16 \times 10^{-15} \\
30\end{array}$ & - \\
\hline & $\mathrm{Cl}^{252}$ & $\begin{array}{l}\text { Maximum yield } \\
\text { Time }^{\circ}\end{array}$ & 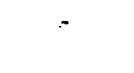 & - & - & $\begin{array}{c}1.20 \times 10^{-15} \\
16\end{array}$ & $\begin{array}{c}2.51 \times 10^{-14} \\
11\end{array}$ & $\begin{array}{c}1.29 \times 10^{-13} \\
8\end{array}$ \\
\hline
\end{tabular}

* Indicates time to attain maximum yield in units of $\sec \times 10^{6}\left(10^{7} \mathrm{sec} \approx 4\right.$ months).

The data of Table II indicate that there are no cases in which a pronounced "peaking" of the maximum yield occurs as in the $\mathrm{Am}^{241}-\mathrm{Cm}^{242}$ case; that is, there are no situations in which an intermediate flux is markedly more effective in producing maximum yield than fluxes of lower or higher intensity. A small peak effect does occur with $\mathrm{Pu}^{242}$ targets but would be of little practical importance. The peak in this case is probably because the first capture product, $\mathrm{Pu}^{243}$, has a relatively large destruction cross section so that losses at higher fluxes to fission products and $\mathrm{Pu}^{244}$ production begin seriously to compete with the decay to $\mathrm{Am}^{243}$ as follows:

$$
\mathrm{Pu}^{242} \stackrel{\sigma_{\mathrm{c}}=50}{\longrightarrow} \mathrm{Pu}_{\sigma_{\mathrm{D}}}^{243} \underset{\mathrm{a}}{\stackrel{4.98 \mathrm{~h}}{\longrightarrow}} \mathrm{Am}^{243}-\rightarrow-\text { Further buildup. }
$$

With other desired products up to mass 252 , the general tendency is for yields to level off at a flux of about $10^{15}$, while yields of products of heavier mass still continue to increase in the $10^{15}-10^{16}$ range. Where leveling of yield does occur, the time to reach maximum yield varies inversely with the flux; that is, the yields are nvt-dependent.

Other minor variations seen in Table II are probably due to slight changes in assumed cross sections and half-lives between the individual reports. Certainly none of the differences would be of major concern from a production point of view. 


\section{REFERENCES}

1. John Milsted, P. R. Fields, and D. N. Metta, Nucl. Applic. 1, 136-144 (Apr 1965); Calculations of Yields of Products in High Flux Neutron Irradiations of Heavy Element Samples, USAEC Report ANL-6756 (Aug 1963).

2. D. C. Stewart, R. W. Anderson, and John Milsted, The Production of Curium by Neutron Irradiation of Am ${ }^{241}$, USAEC Report ANL-6933 (Nov 1964).

3. D. C. Stewart, R. W. Anderson, and John Milsted, Data Relating to the Production of Transcurium Elements in High Neutron Fluxes, USAEC Report ANL-6932 (Sept 1964).

4. H. Bateman, Proc. Camb. Phil. Soc. 15, 423 (1910). 